\title{
International Consensus on Antineutrophil Cytoplasm Antibodies Testing in Eosinophilic Granulomatosis with Polyangiitis
}

Citation for published version (APA):

Moiseev, S., Bossuyt, X., Arimura, Y., Blockmans, D., Csernok, E., Damoiseaux, J., Emmi, G., FloresSuarez, L. F., Hellmich, B., Jayne, D., Jennette, J. C., Little, M. A., Mohammad, A. J., Moosig, F., Novikov, P., Pagnoux, C., Radice, A., Sada, K., Segelmark, M., ... European EGPA Study Group (2020). International Consensus on Antineutrophil Cytoplasm Antibodies Testing in Eosinophilic Granulomatosis with Polyangiitis. American Journal of Respiratory and Critical Care Medicine, 202(10), 1360-1372. https://doi.org/10.1164/rccm.202005-1628SO

Document status and date:

Published: 15/11/2020

DOI:

10.1164/rccm.202005-1628SO

Document Version:

Publisher's PDF, also known as Version of record

\section{Document license:}

Taverne

Please check the document version of this publication:

- A submitted manuscript is the version of the article upon submission and before peer-review. There can be important differences between the submitted version and the official published version of record.

People interested in the research are advised to contact the author for the final version of the publication, or visit the DOI to the publisher's website.

- The final author version and the galley proof are versions of the publication after peer review.

- The final published version features the final layout of the paper including the volume, issue and page numbers.

Link to publication

\footnotetext{
General rights rights.

- You may freely distribute the URL identifying the publication in the public portal. please follow below link for the End User Agreement:

www.umlib.nl/taverne-license

Take down policy

If you believe that this document breaches copyright please contact us at:

repository@maastrichtuniversity.nl

providing details and we will investigate your claim.
}

Copyright and moral rights for the publications made accessible in the public portal are retained by the authors and/or other copyright owners and it is a condition of accessing publications that users recognise and abide by the legal requirements associated with these

- Users may download and print one copy of any publication from the public portal for the purpose of private study or research.

- You may not further distribute the material or use it for any profit-making activity or commercial gain

If the publication is distributed under the terms of Article 25fa of the Dutch Copyright Act, indicated by the "Taverne" license above, 


\title{
International Consensus on Antineutrophil Cytoplasm Antibodies Testing in Eosinophilic Granulomatosis with Polyangiitis
}

\author{
Sergey Moiseev ${ }^{1}$, Xavier Bossuyt ${ }^{2}$, Yoshihiro Arimura ${ }^{3}$, Daniel Blockmans ${ }^{4}$, Elena Csernok ${ }^{5}$, Jan Damoiseaux ${ }^{6}$,
} Giacomo Emmi ${ }^{7}$, Luis Felipe Flores-Suárez ${ }^{8}$, Bernhard Hellmich ${ }^{9}$, David Jayne ${ }^{10}$, J. Charles Jennette ${ }^{11}$, Mark A. Little ${ }^{12}$, Aladdin J. Mohammad ${ }^{13,14}$, Frank Moosig ${ }^{15}$, Pavel Novikov ${ }^{1}$, Christian Pagnoux ${ }^{16}$, Antonella Radice ${ }^{17}$, Ken-ei Sada ${ }^{18}$, Mårten Segelmark ${ }^{19}$, Yehuda Shoenfeld ${ }^{20,21}$, Renato A. Sinico ${ }^{22}$, Ulrich Specks ${ }^{23}$, Benjamin Terrier" Athanasios G. Tzioufas ${ }^{25}$, Augusto Vaglio ${ }^{26}$, Ming-Hui Zhao ${ }^{27}$, and Jan Willem Cohen Tervaert ${ }^{28,29}$; on behalf of the European EGPA Study Group

\begin{abstract}
${ }^{1}$ Tareev Clinic of Internal Diseases, Sechenov First Moscow State Medical University, Moscow, Russia; ${ }^{2}$ Laboratory Medicine, University Hospitals Leuven, and Department of Microbiology, Immunology and Transplantation, KU Leuven, Leuven, Belgium; ${ }^{3}$ Department of Nephrology and Rheumatology, Kyorin University School of Medicine, Tokyo, Japan; ${ }^{4}$ Clinical Department of General Internal Medicine Department, Research Department of Microbiology and Immunology, Laboratory of Clinical Infectious and Inflammatory Disorders, University Hospitals Leuven, Leuven, Belgium; ${ }^{5}$ Department of Internal Medicine, Rheumatology and Immunology, Vasculitis-Center Tübingen-Kirchheim, Medius Klinik Kirchheim, University of Tübingen, Kirchheim-Teck, Germany; ${ }^{6}$ Central Diagnostic Laboratory, Maastricht University Medical Centre, Maastricht, the Netherlands; ${ }^{7}$ Department of Experimental and Clinical Medicine, University of Firenze, Careggi Hospital, Firenze, Italy; ${ }^{8}$ Primary Systemic Vasculitides Clinic, Instituto Nacional de Enfermedades Respiratorias, Mexico City, Mexico; ${ }^{9}$ Klinik für Innere Medizin, Rheumatologie und Immunologie, Vaskulitis-Zentrum Süd, Medius Kliniken, Akademisches Lehrkrankenhaus der Universität Tübingen, Kirchheim-unter-Teck, Germany; ${ }^{10}$ Department of Medicine, University of Cambridge, Cambridge, United Kingdom; ${ }^{11}$ Division of Nephropathology, School of Medicine, University of North Carolina, Chapel Hill, North Carolina; ${ }^{2}$ Trinity Health Kidney Centre, Trinity Translational Medicine Institute, Dublin, Ireland; ${ }^{13}$ Department of Rheumatology, Skåne University Hospital, Lund, Sweden, Vasculitis; ${ }^{14}$ Lupus Clinic, Addenbrooke's Hospital, Cambridge University Hospitals, Cambridge, United Kingdom; ${ }^{15}$ Rheumazentrum, Schleswig-Holstein Mitte, Neumünster, Germany; ${ }^{6}$ Division of Rheumatology, Department of Medicine, Mount Sinai Hospital Vasculitis Clinic, University of Toronto, Toronto, Ontario, Canada; ${ }^{17}$ Microbiology and Virology Institute, ASST Santi Paolo e Carlo, San Carlo Borromeo Hospital, Milan, Italy; ${ }^{18}$ Department of Nephrology, Rheumatology, Endocrinology and Metabolism, Okayama University Graduate School of Medicine, Dentistry and Pharmaceutical Sciences, Okayama, Japan; ${ }^{19}$ Department of Clinical Sciences, Lund University, Department of Nephrology and Rheumatology, Skane University Hospital, Lund, Sweden; ${ }^{20}$ Zabludowicz Center for Autoimmune Diseases, Sheba Medical Center, Tel Hashomer, Sackler Faculty of Medicine, Tel Aviv University, Tel Aviv, Israel; ${ }^{21}$ Sechenov First Moscow State Medical University, Moscow, Russia; ${ }^{22}$ Department of Medicine and Surgery, Università degli Studi di Milano-Bicocca, Monza, Italy; ${ }^{23}$ Division of Pulmonary and Critical Care Medicine, Mayo Clinic, Rochester, Minnesota; ${ }^{24}$ Department of Internal Medicine, National Referral Center for Rare Systemic and Autoimmune Diseases, Hôpital Cochin, Paris, France; ${ }^{25}$ Department of Pathophysiology, Medical School, National and Kapodistrian University of Athens, Athens, Greece; ${ }^{26}$ Department of Biomedical Experimental and Clinical Sciences “Mario Serio", University of Firenze, Meyer Children's Hospital, Firenze, Italy; ${ }^{27}$ Renal Division, Peking University First Hospital, Key Laboratory of Renal Disease, Ministry of Health of China, Key Laboratory of CKD Prevention and Treatment, Ministry of Education of China, Peking-Tsinghua Centre for Life Sciences, Beijing, China; ${ }^{28}$ Department of Medicine, Division of Rheumatology, University of Alberta, Edmonton, Alberta, Canada; and ${ }^{29}$ Maastricht University, Maastricht, the Netherlands
\end{abstract}

ORCID ID: 0000-0002-7232-4640 (S.M.)

\begin{abstract}
An international consensus on antineutrophil cytoplasm antibodies (ANCA) testing in eosinophilic granulomatosis with polyangiitis (EGPA) is presented. ANCA, specific for myeloperoxidase (MPO), can be detected in 30-35\% of patients with EGPA. MPO-ANCA should be tested with antigen-specific immunoassays in any patient with eosinophilic asthma and clinical features suggesting EGPA, including constitutional symptoms; purpura; polyneuropathy; unexplained heart, gastrointestinal, or kidney disease; and/or pulmonary infiltrates or hemorrhage. A positive MPO-ANCA result contributes to the diagnostic workup for EGPA. Patients with MPO-ANCA-associated EGPA have vasculitis features,
\end{abstract}

such as glomerulonephritis, neuropathy, and skin manifestations, more frequently than patients with ANCA-negative EGPA. However, the presence of MPO-ANCA is neither sensitive nor specific enough to identify whether a patient should be subclassified as having "vasculitic" or "eosinophilic" EGPA. At present, ANCA status cannot guide treatment decisions, that is, whether cyclophosphamide, rituximab, or mepolizumab should be added to conventional glucocorticoid treatment. In EGPA, monitoring of ANCA is only useful when MPO-ANCA was tested positive at disease onset.

Keywords: eosinophilic granulomatosis with polyangiitis; ANCA; vasculitis; consensus

(Received in original form May 6, 2020; accepted in final form June 23, 2020)

A list of European EGPA Study Group members not included in the list of authors may be found before the beginning of the REFERENCES.

Supported by the Russian Academic Excellence Project 5-100 (S.M. and P.N.).

Am J Respir Crit Care Med Vol 202, Iss 10, pp 1360-1372, Nov 15, 2020

Copyright (C) 2020 by the American Thoracic Society

Originally Published in Press as DOI: 10.1164/rccm.202005-1628SO on June 25, 2020

Internet address: www.atsjournals.org 


\section{Table of Contents Methods \\ Prevalence of ANCA in Patients with EGPA \\ Clinical Value of ANCA in EGPA}

\author{
MPO-ANCA as a Classification \\ Criterion for EGPA \\ MPO-ANCA as a Guide for Treatment \\ Decisions
}

\section{ANCA Monitoring Conclusions}

Antineutrophil cytoplasm antibodies (ANCA) were first described more than 50 years ago. During the last four decades, it was discovered that these antibodies are a distinctive laboratory feature of glomerulonephritis and vasculitis (1-3). ANCA are detected in most patients with granulomatosis with polyangiitis (GPA) and microscopic polyangiitis (MPA), and in some patients with eosinophilic granulomatosis with polyangiitis (EGPA). ANCA can be detected in sera by indirect immunofluorescence (IIF) and/or solid-phase immunoassays, such as ELISA, fluoroenzyme immunoassay, chemiluminescence immunoassay, laser bead immunoassay, or dot/line blot. In patients with ANCAassociated vasculitis (AAV), IIF reveals two major patterns: either cytoplasmic ANCA (C-ANCA) or perinuclear ANCA (P-ANCA), whereas immunoassays reveal antibodies specific for proteinase-3 (PR3-ANCA) and myeloperoxidase (MPO-ANCA).

In 2017, a revised international consensus on ANCA testing proposed that high-quality immunoassays can be used as the primary screening method for patients suspected of having GPA and MPA (1). A multicenter European Vasculitis Society (EUVAS) study showed a large variability between two IIF methods and a good diagnostic performance of PR3-ANCA and MPO-ANCA immunoassays (2, 3). The 2017 revised international consensus did not include EGPA. The current document is a follow-up on the revised consensus statement (1) and focuses on the clinical and diagnostic value of ANCA testing in patients suspected of having EGPA.

\section{Methods}

This consensus statement was prepared by a group of experts. References for this consensus statement were identified through searches of PubMed, Embase, and Scopus for articles published from January 1951 to January 2020 by use of the terms "eosinophilic granulomatosis with polyangiitis," "Churg-Strauss syndrome," and "eosinophilic vasculitis." Additional publications were identified in the references of the available articles. Articles resulting from these searches and relevant references cited in those articles were reviewed. Articles published in English, French, and German were included. The resulting manuscript was distributed by e-mail to experts from four continents, including rheumatologists, pulmonologists, immunologists, nephrologists, and specialists in laboratory medicine, selected based on their expertise and knowledge in clinical and laboratory aspects of ANCA-associated vasculitides and ANCA testing. All contributors approved the final document and voted for each statement using a 5-point Likert scale (strongly disagree, disagree, uncertain, agree, and strongly agree). The definition for consensus included percentage agreement of at least $80 \%$ and the median score $\geqslant 4$.

\section{Prevalence of ANCA in Patients with EGPA}

In 1989, Harrison and colleagues studied different cytoplasmic patterns when testing for ANCA by IIF in patients with vasculitis. Three patients with Churg-Strauss syndrome (or EGPA according to the current nomenclature) had atypical cytoplasmic staining (4). Cohen Tervaert and colleagues studied the clinical associations of MPO-ANCA in 53 patients and found that 6 patients (11\%) had biopsyproven EGPA (5). In addition, many patients that tested positive for MPO-ANCA had symptoms suggestive of EGPA without fulfilling the classification criteria for EGPA (5, 6). PR3-ANCA and MPO-ANCA were found to be specific for GPA, MPA, and/or EGPA. Although the majority $(>90 \%)$ of patients with GPA and MPA are ANCA positive, in EGPA this is $<50 \%$. Even patients with EGPA with biopsy-proven necrotizing arteritis could test ANCA negative $(5,7)$. The occurrence of MPO-ANCA in patients with EGPA suggested that EGPA belongs to the group of vasculitides encompassing GPA and MPA (8).

To evaluate the prevalence of ANCA in patients with EGPA, we selected 24 studies that included at least 30 patients and were published in peer-reviewed journals (Table 1) (9-32). In most studies, sera from patients were initially screened by IIF. MPO-ANCA and PR3-ANCA were subsequently assessed by antigen-specific immunoassays only in patients in whom ANCA had been detected by IIF. The reported prevalence of ANCA positivity was variable, ranging from $14.6 \%$ to $73.0 \%$ as assessed by IIF and from $14.6 \%$ to $60.6 \%$ as assessed by ELISA. The median frequencies were $33.0 \%$ and $30.6 \%$ for IIF and ELISA, respectively. The reported high variability of ANCA prevalence is probably due to the small number of patients in some studies, to selection bias, to the different classification criteria used for EGPA, and to the various methods used to test for ANCA (12). For example, in one study, the prevalence of ANCA positivity depended largely on the different organ involvement of patients and ranged from $0-12.5 \%$ in pulmonary patients to $90-100 \%$ in patients with renal disease (12). However, the median frequency of ANCA positivity in the selected studies was similar to that in the largest study, in which ANCA were detected by IIF and specific immunoassays in $33.0 \%$ and $30.7 \%$ of 534 patients with EGPA, respectively (32).

The studies usually reported a higher occurrence of ANCA by IIF compared with that by ELISA, because solid-phase immunoassays could not confirm the

Author Contributions: All authors contributed substantially to the interpretation of data, critically revised the manuscript for important intellectual content, approved the final version, and agreed to be accountable for all aspects of the work in ensuring that questions related to the accuracy or integrity of any part of the work are appropriately investigated and resolved.

Correspondence and requests for reprints should be addressed to Sergey Moiseev, M.D., Tareev Clinic of Internal Diseases, Sechenov First Moscow State Medical University, Rossolimo, 11/5, Moscow 119435, Russia. E-mail: avt420034@yahoo.com. 


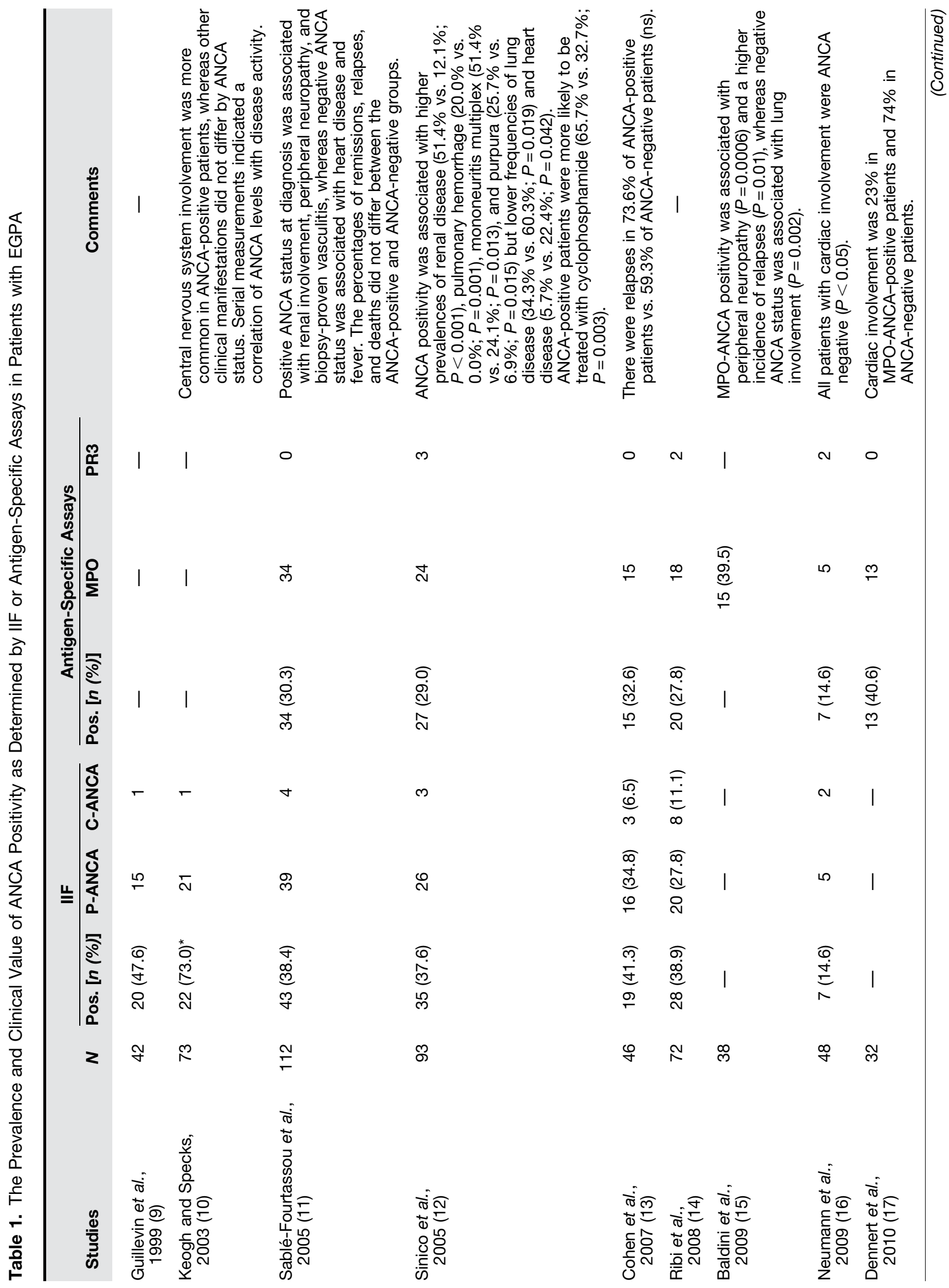




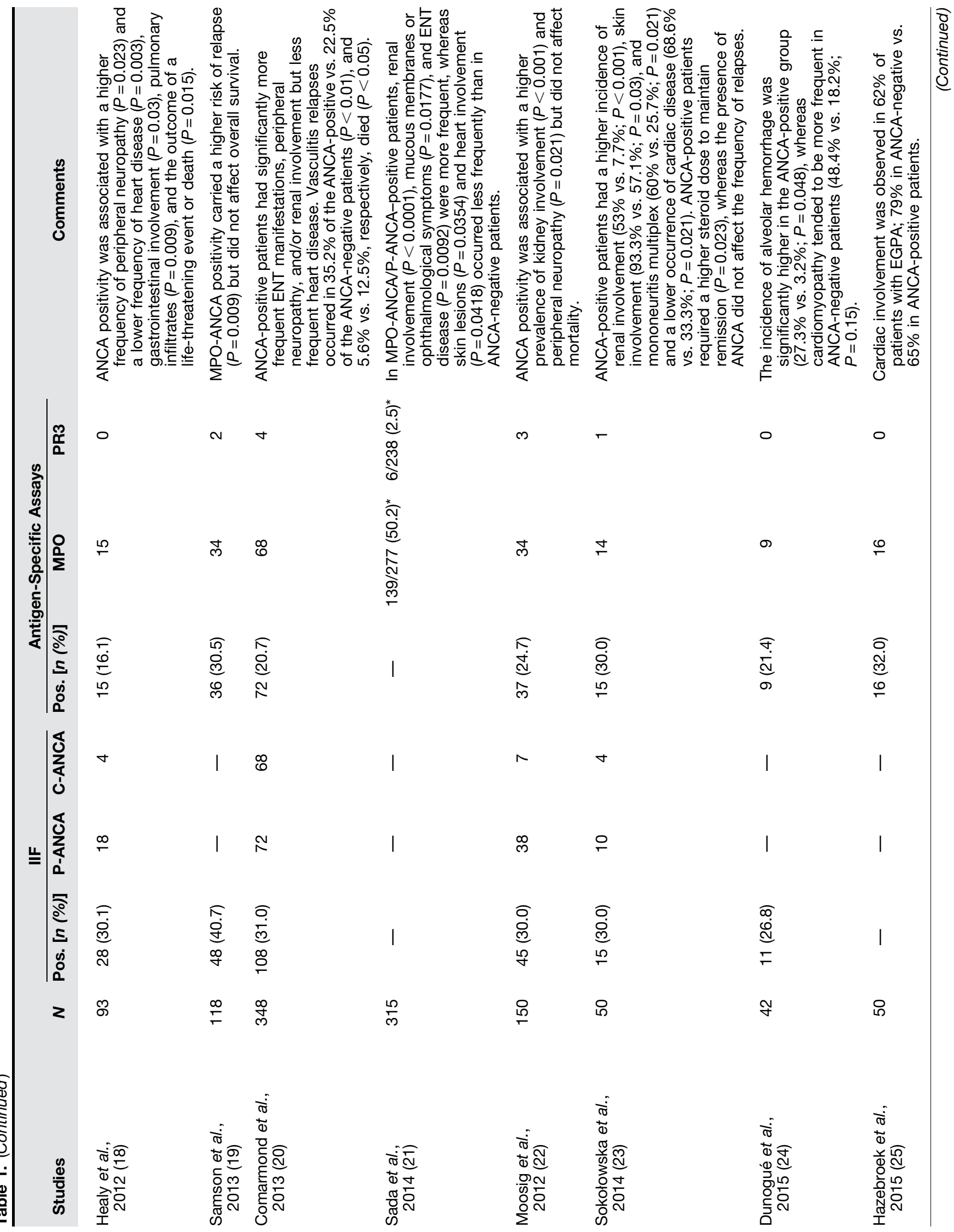




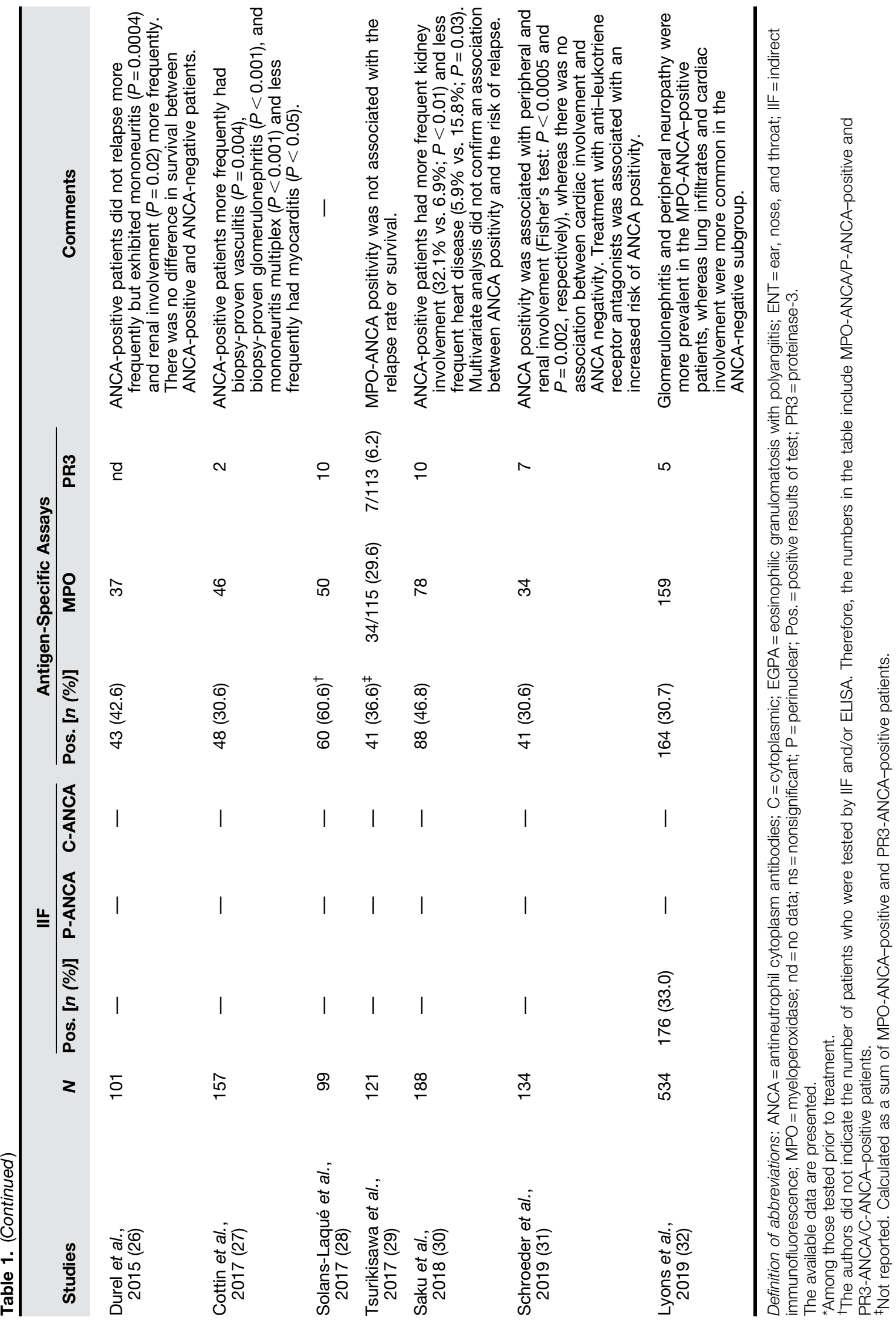


presence of MPO-ANCA or PR3-ANCA in a small proportion of IIF-positive patients showing P-ANCA, C-ANCA, or atypical ANCA patterns. Sinico and colleagues compared the performance of IIF on ethanol-fixed granulocytes and antigenspecific ELISA in 93 patients with EGPA (12). ANCA were detected by IIF in 35 of 93 patients $(37.6 \%)$ tested at the time of diagnosis. Two of 26 P-ANCA-positive samples were negative for MPO-ANCA by ELISA, whereas MPO-ANCA was detected in 6 patients with C-ANCA or atypical ANCA patterns. These latter six samples showed an atypical cytoplasmic pattern when tested in the central laboratory, which contrasted with the reported finding in the local laboratory. These data are in accordance with the results of a multicenter EUVAS evaluation of IIF versus antigenspecific immunoassays in patients with GPA and MPA, underscoring the high variability in results obtained by IIF in different laboratories $(2,3)$.

When ANCA was found positive in EGPA, the antibodies are in most cases directed against MPO rather than against PR-3. The proportion of MPO-ANCA among all patients with ANCA-positive EGPA ranged from $71.4 \%$ to $100 \%$ with a median of $93.3 \%$. In the two largest studies that included 348 and 534 patients with EGPA, MPO-ANCA were detected in $94.4 \%$ and $97.0 \%$, respectively, of the patients that tested positive for ANCA by ELISA $(20,32)$. The remaining patients tested positive for PR3-ANCA.

$\mathrm{P}$-ANCA is found not only in patients with MPO-ANCA but also in patients with antibodies to lactoferrin, elastase, cathepsin G, lysozyme, and other antigens (33). Recently, antilactoferrin antibodies were detected in patients with EGPA $(4 / 19,21.1 \%)$ but not in patients with GPA or MPA. Antilactoferrin antibodies were associated with disease activity (34). However, because antilactoferrin antibodies can be found in a wide variety of autoimmune disorders, further studies are needed to clarify their clinical value in EGPA.

Mukherjee and colleagues reported polyclonal ANCA reactivity in sputum samples of patients with EGPA, irrespective of the presence of MPO-ANCA in the sera of these patients (35). Sputum ANCA positivity was associated with severe respiratory symptoms, and immunoglobulins from ANCA-positive sputum triggered extensive extracellular trap formations from both neutrophils and eosinophils in vitro, indicating possible pathogenicity of detected IgG autoantibodies. Detection of ANCA in sputum probably precedes serum ANCA positivity and may identify a subset of patients with eosinophilic asthma who are at increased risk of developing EGPA in the future.

In conclusion, ANCA can be detected by IIF or antigen-specific immunoassays in approximately $30-35 \%$ of patients with EGPA. Of the patients with EGPA that test positive for ANCA, most (up to 90-100\%) have MPO-ANCA. The results of IIF may be inconsistent in a proportion of patients with EGPA when tested in different laboratories or on different substrates.

\section{Clinical Value of ANCA in EGPA}

ANCA status defines two subsets of patients with EGPA who have different clinical disease phenotypes (Figure 1). Most studies that evaluated an association between ANCA positivity and predominant clinical features of EGPA showed that ANCApositive patients were more likely to have vasculitis manifestations, such as glomerulonephritis, peripheral neuropathy, alveolar hemorrhage, or purpura, and less frequently had heart disease or granulomatous lung involvement compared with ANCA-negative patients (Table 1). For example, in the study by Lyons and colleagues (534 patients with EGPA, including $159 \mathrm{MPO}-\mathrm{ANCA}$ positive), the presence of MPO-ANCA was associated with a higher occurrence of peripheral neuropathy (78.6\% vs. $57.1 \%)$ and glomerulonephritis (28.9\% vs. $9.4 \%)$ and a lower frequency of lung infiltrates $(45.3 \%$ vs. $61.4 \%)$ and cardiomyopathy (14.5\% vs. $30.4 \%$ ) (32). These divergent clinical associations remained statistically significant after adjustment for country of origin.

Similar associations were reported by Comarmond and colleagues in 348 patients with EGPA (20). In this study, biopsyproven vasculitis was more frequent in ANCA-positive patients than in ANCAnegative patients $(77.4 \%$ vs. $48.8 \%$, $P=0.01)$, whereas granulomatous and eosinophilic infiltrates occurred with a similar frequency in the two groups. ANCA-positive patients were more likely to develop vasculitis relapses and less likely to die than ANCA-negative patients. The higher mortality rate associated with ANCA-negative status was probably related to a higher occurrence of cardiomyopathy (25) that was the main independent predictor of death in patients with EGPA on multivariable analysis. However, most other studies did not confirm that ANCA-negative status affects overall

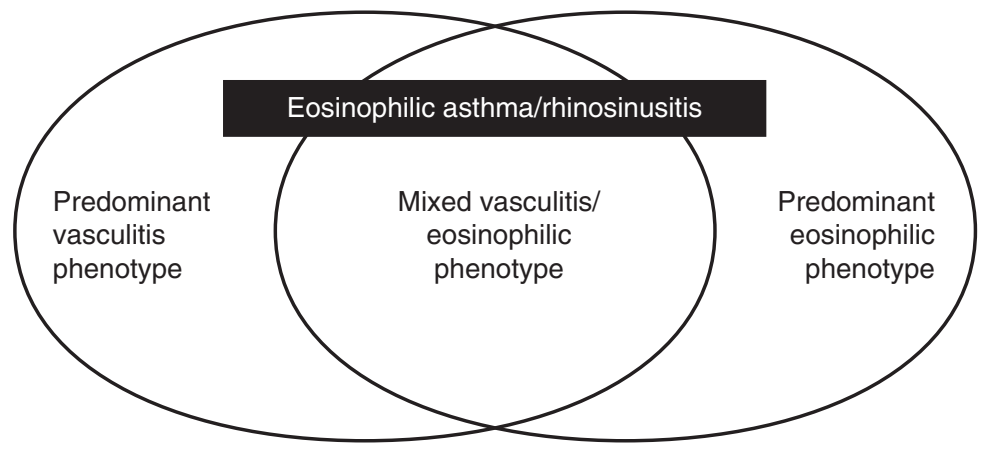

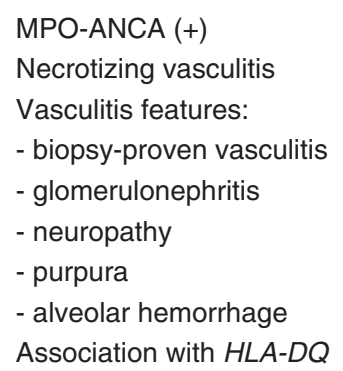

MPO-ANCA (+)

ecrotizing vasculitis

- glomerulonephritis

- neuropathy

- purpura

Association with $H L A-D Q$
MPO-ANCA (-)

Eosinophilic and

granulomatous inflammation

Tissue infiltration:

- lung infiltrates

- cardiomyopathy

- gastrointestinal disease

Association with GPA33 and IL5/IRF1

Figure 1. Clinical phenotypes and genetic features of eosinophilic granulomatosis with polyangiitis. MPO-ANCA = myeloperoxidase-antineutrophil cytoplasm antibodies. 
survival $(11,19,22,26,29)$. These differences may reflect differences in methods used to perform cardiac screening in these patients (17) and time from disease onset to diagnosis in patients with severe cardiac involvement. Also, several studies reported no association between ANCA positivity and a higher relapse rate in EGPA $(23,26,29,30)$. PR3-ANCA are found in only $1-2 \%$ of patients with EGPA $(12,20)$, and little is known about their association with clinical manifestations and outcome.

The pathogenic role of MPO-ANCA in vasculitis was shown in experimental studies. In MPO-immunized rats, pulmonary artery perfusion with polymorphonuclear leukocytes lysosomal enzymes resulted in extensive lung injury with granuloma-like lesions and giant cell formation (36), whereas the transfer of splenocytes from MPO-deficient mice immunized with murine MPO led to the development of glomerulonephritis and pulmonary capillaritis (37).

However, available evidence suggests that vasculitis in patients with EGPA can occur in both the presence and absence of ANCA (7). In the study by Cottin and colleagues, definite clinical or pathologic features of vasculitis were found in $28 \%$ of ANCA-negative patients and were absent in $29 \%$ of MPO-ANCA-positive patients. These data suggest that MPO-ANCA cannot be used as a unique surrogate of systemic vasculitis (27).

A recent genome-wide association study that tested 9.2 million genetic variants in 534 cases and 6,688 controls provided additional evidence for genetic distinctions between MPO-ANCA-positive and ANCAnegative EGPA (32). Analysis of MPOANCA-positive versus ANCA-negative cases revealed a genome-wide significant association at $r s 17212014$ in the HLA-DQ region for MPO-ANCA-positive status, whereas ANCA-negative status alone was associated with variants at the GPA33 and IL5/IRF1 loci. ANCA-negative EGPA was more genetically similar to asthma than MPO-ANCA-positive EGPA. The authors suggested that MPO-ANCA-positive EGPA is an eosinophilic autoimmune disease sharing clinical features and an $H L A-D Q$ association with MPO-

ANCA-associated vasculitis, whereas

ANCA-negative EGPA may instead have a mucosal barrier dysfunction origin.

Despite the clinical, histological, and genetic differences between ANCA-positive and ANCA-negative EGPA, few studies have reported whether distinct pathophysiological mechanisms or molecular pathways operate in these two subsets. Reports investigating the clinical or prognostic significance of circulating biomarkers (e.g., eosinophil counts, eotaxin3, IgG4) either did not assess or failed to detect associations between such parameters and the ANCA status (38-41). In a recent review, Chaigne and colleagues speculated that vasculitis phenotype of EGPA may be mediated by neutrophils, neutrophil extracellular traps, and B lymphocytes, whereas eosinophils may drive the nonvasculitis phenotype (42). Specific data on neutrophil extracellular traps in EGPA are lacking, although a link between enhanced netosis and MPOANCA-associated vasculitis was shown in animal models $(43,44)$. A potential role for $B$ lymphocytes in the pathogenesis of vasculitis in EGPA is suggested by both clinical data and efficacy of rituximab in a proportion of patients with EGPA.

Eosinophils release toxic products such as eosinophilic cationic protein and eosinophil-derived neurotoxin that may directly damage nerve fibers or endomyocardial tissue. Moreover, eosinophils may induce ischemic damage through occlusion of vessels or activation of the clotting cascade.

In MPO-ANCA-negative patients, sural nerve biopsy findings showed that large numbers of eosinophils occluded epineural vessels, whereas in sural nerve biopsies of patients with MPOANCA-associated EGPA, necrotizing vasculitis of epineural vessels was more frequently found (45). On the basis of these results, Nishi and colleagues recently postulated two distinct pathophysiological mechanisms in the two different phenotypes: in the vasculitis subtype, ischemia and tissue damage is due to necrotizing vasculitis, whereas in the nonvasculitis phenotype, intraluminal eosinophils occlude blood vessels, resulting in ischemia and eosinophil-associated tissue damage.

In summary, clinical and genetic evidence supports the use of ANCA testing to define two distinctive subsets of EGPA. However, the clinical value of ANCA positivity should not be overestimated, given a significant overlap between two clinical phenotypes. Clinical and/or pathologic features of vasculitis, such as glomerulonephritis or peripheral neuropathy, can be observed in both patients with ANCA-negative EGPA and those with ANCA-positive EGPA. Moreover, some disease manifestations including peripheral neuropathy or cardiomyopathy could be due to both vasculitis and eosinophilic infiltration (46). Given the primacy of the association of genetic polymorphisms with ANCA serotype, future disease classifications based on serotype will have an influence on the subclassification of EGPA, with the possibility that PR3-ANCA and MPOANCA-positive EGPA will become eosinophilic variants of PR3-ANCA and MPO-ANCA-positive AAV.

\section{MPO-ANCA as a Classification Criterion for EGPA}

In 1984, Lanham and colleagues proposed the first criteria for EGPA, which included asthma, blood eosinophilia, and evidence for vasculitis involving two or more organs (47). The 1990 American College of Rheumatology classification criteria included asthma, blood eosinophilia $>10 \%$, neuropathy, nonfixed pulmonary infiltrates, paranasal sinus abnormality, and biopsy containing a blood vessel with extravascular eosinophils. The presence of at least four of these six criteria yielded a sensitivity of $85 \%$ and a specificity of $99.7 \%$ (48). In 2007, the European Medicines Agency algorithm based on American College of Rheumatology criteria and Chapel Hill Consensus Conference (CHCC) definitions provided a stepwise approach for classifying patients with AAV into the disease types for the purposes of epidemiological studies (49). The presence of ANCA was included in this algorithm.

The presence of ANCA was first indicated in the definition of EGPA at the 2012 CHCC, which stated that ANCA is more frequent when glomerulonephritis is present (50). However, CHCC was a nomenclature system specifying the name that should be used for a defined disease process. CHCC definitions were not intended as classification criteria and cannot be used for diagnostic purposes. Because histological data may not be available for all patients with suspected vasculitis, surrogates for vasculitis, such as clinical and laboratory findings suggestive 
of vasculitis in the absence of histologic evidence, may be used (50).

Over the last few years, classification schemes for EGPA continued to evolve and incorporated ANCA testing as a valuable diagnostic aid. In 2015, the EGPA Consensus Task Force issued recommendations for evaluation and management of patients with EGPA stating that, in the clinical context of asthma and eosinophilia, MPO-ANCA positivity is highly suggestive for EGPA, but ANCA negativity does not rule out this diagnosis (51). Therefore, ANCA testing with IIF and ELISA was recommended for patients with suspected EGPA. The Task Force also stated that the presence of a C-ANCA pattern or PR3-ANCA is unusual for EGPA, and diagnosis should be critically reviewed in this setting.

Recently, the "Groupe d'Etudes et de Recherche sur les Maladies Orphelines Pulmonaires" and the "European Respiratory Society Task Force" suggested that EGPA with genuine vasculitis features (absent in at least $40 \%$ of patients with EGPA) should be differentiated from an eosinophilic tissue infiltrate phenotype based on the presence of definite vasculitis features (e.g., biopsy-proven necrotizing vasculitis of any organ), strong surrogate markers of vasculitis, such as mononeuritis multiplex, and/or the presence of ANCA as determined by antigen-specific assay with at least one extrathoracic non-ear, nose, and throat (ENT) manifestation of disease (27). The Task Force proposed a new terminology for this eosinophilic tissue phenotype, that is, hypereosinophilic asthma with systemic (nonvasculitic) manifestations. Patients with hypereosinophilic asthma with systemic (nonvasculitic) manifestations typically present with asthma, blood eosinophilia $>1.5 \times 10^{9} / \mathrm{L}$, and systemic manifestations but without biopsy-proven vasculitis and/or surrogate markers of vasculitis. Of note, the Task Force acknowledged the clinical value of ANCA as a surrogate of systemic vasculitis when associated with systemic manifestations and recommended testing for ANCA using an antigen-specific assay.

In the MIRRA study (a double-blind randomized placebo-controlled study to investigate the efficacy and safety of mepolizumab in the treatment of EGPA in subjects receiving standard of care therapy), a positive result for antigen-specific ANCA was listed as one of at least two additional features necessary to define eosinophilic asthma as EGPA (52).

New classification criteria for ANCAassociated vasculitides including EGPA have been drafted based on the data from the DCVAS (Diagnostic and Classification of the Systemic Vasculitides) study and are currently undergoing formal review by the American College of Rheumatology and the European League Against Rheumatism (53).

Over the last decade, ANCA testing has become a part of the routine workup of patients with suspected EGPA. The sensitivity of MPO-ANCA detection as a classification criterion for EGPA is low ( 30-35\%). The presence of MPO-ANCA as a vasculitis surrogate should, however, be used only in the right clinical context because antigen-specific ANCA testing can give positive results in various inflammatory disorders mimicking ANCAassociated vasculitis (54).

Importantly, MPO-ANCA in combination with blood eosinophilia and vasculitic symptoms may occur in the setting of cholesterol emboli syndrome. This syndrome occurs in patients with severe atherosclerosis and is associated with acrocyanosis, livedo reticularis, progressive renal failure, and other signs and symptoms (e.g., fever, weight loss, myalgia, leukocytosis, eosinophilia, raised erythrocyte sedimentation rate and C-reactive protein) mimicking vasculitis $(55,56)$.

\section{MPO-ANCA as a Guide for Treatment Decisions}

Glucocorticoids are the cornerstone of therapy for EGPA. Additional immunosuppressive agents (e.g., cyclophosphamide) should be prescribed for patients with life- and/or organthreatening manifestations, such as heart disease, glomerulonephritis, alveolar hemorrhage, or mononeuritis multiplex, and can be considered for selected patients with glucocorticoid dependence or recurrent disease (51). The Five-Factor Score (FFS) is frequently used to decide whether cytotoxic drugs are indicated $(\mathrm{FFS} \geqslant 1)$ or not $(\mathrm{FFS}=0)$. Importantly, however, this index does not include alveolar hemorrhage and/or mononeuritis multiplex, conditions that may have a severe impact on function in patients with EGPA (57). In several studies, patients with ANCA-positive EGPA were more likely to be treated with cyclophosphamide (12) or required a higher glucocorticoid dose to maintain remission (53). However, MPOANCA positivity alone does not justify more intensive immunosuppressive treatment.

Other treatment options for EGPA include rituximab, an anti-CD20 monoclonal antibody directed against B cells, and mepolizumab, a humanized monoclonal antibody that targets IL-5. Currently, rituximab is frequently used for both remission induction and maintenance therapy for GPA and MPA, based on the results of randomized controlled clinical trials (58-60). The efficacy of rituximab in EGPA was not evaluated in clinical trials but was shown in case reports and case series (61-64). The rationale for using rituximab in EGPA comes from the known overlap between various ANCA-associated vasculitides, that is, ANCA positivity and vasculitis features (although less common in EGPA than in GPA/MPA) (63), and the ability of rituximab to reduce T-cell-derived production of IL-5 that induces stimulation and maturation of eosinophils (65).

Mohammad and colleagues presented data on 41 patients with refractory, relapsing, or new-onset EGPA (44\% ANCA positive) treated with rituximab in four vasculitis centers (62). Rituximab administration resulted in the improvement of disease activity in $90 \%$ of patients at 12 months and the reduction of prednisolone dose, although only $6 \%$ of patients were able to discontinue glucocorticoids completely. ANCA-positive patients were more likely to achieve remission when on rituximab than ANCA-negative patients ( $80 \%$ vs. $38 \%$; $P=0.013$ ).

In a retrospective study, Teixeira and colleagues evaluated the longer-term efficacy and safety of rituximab in 69 patients with EGPA (34.8\% ANCA positive) treated in a single tertiary center (63). By 24 months, rituximab administration was associated with a complete or partial response in $93 \%$ of patients and a reduction in the median prednisolone dose from 12.5 to $5 \mathrm{mg} / \mathrm{d}$. Nevertheless, asthma and ENT relapse rates were high despite repeat rituximab dosing. At 6 and 12 months, more ANCA-positive patients were in remission compared with ANCA-negative patients $(29.2 \%$ vs. $13.3 \%$ and $34.8 \%$ vs. $23.1 \%$, respectively), but the differences between the two groups did not reach 
statistical significance. The median time to remission was shorter in the ANCApositive group than in the ANCA-negative group (15 vs. 24 mo; $P=0.02$ ). Moreover, ANCA-positive patients had a longer asthma/ENT relapse-free survival time than ANCA-negative patients $(P=0.04)$. Although there was no difference in the vasculitis relapse rate between the two subsets, none of the 11 patients with glomerulonephritis relapsed during treatment with rituximab, and skin flares were usually described as erythematous and not vasculitic with purpura, suggesting that rituximab treatment may be better in preventing vasculitis relapses.

Emmi and colleagues also found no statistically significant differences in relapse rates or time-to-relapse between patients with ANCA-positive EGPA and those with

ANCA-negative EGPA $(n=15)$ who achieved remission following rituximab induction. Scheduled maintenance with rituximab significantly reduced relapse rate compared with rituximab given "on demand" for relapse (66).

According to the EGPA Consensus Task Force recommendations, rituximab administration may be reasonable for patients with ANCA-positive EGPA with renal involvement or severe refractory disease, despite conventional therapy, for whom traditional cytotoxic agents are contraindicated or undesirable (51). Two prospective trials by the French Vasculitis Study Group are currently investigating rituximab as both induction (ClinicalTrials NCT02807103) and maintenance (ClinicalTrials NCT03164473) therapy of EGPA.

Table 2. Recommendations for ANCA Testing in EGPA

\section{Recommendations}

Recommendation 1

A gating policy for requesting an ANCA test and adherence to clinical indications for ANCA testing (Box 1 ) is advisable.

Recommendation 2

ANCA testing in EGPA should be conducted, as with GPA and MPA, according to a revised 2017 international consensus: high-quality antigen-specific assays for MPO-ANCA and PR3-ANCA should be used as the primary screening method for ANCA.

Recommendation 3

A diagnosis of EGPA cannot be excluded on the basis of negative MPO-ANCA results.

Recommendation 4

A positive MPO-ANCA result only contributes to the diagnostic workup for EGPA and is not diagnostic by itself.

Recommendation 5

A positive MPO-ANCA result is neither sensitive nor specific enough to identify the vasculitis phenotype of EGPA, although MPO-ANCA positivity is associated with a higher occurrence of vasculitis features.

Recommendation 6

ANCA status by itself cannot guide treatment decisions, such as addition of cyclophosphamide, rituximab, or mepolizumab to conventional treatment with glucocorticoids.

Recommendation 7

The result of serial ANCA measurements in patients with MPO-ANCA-associated EGPA (persistence, rise, or reappearance of MPO-ANCA) justifies more frequent clinical assessment.

Definition of abbreviations: ANCA = antineutrophil cytoplasm antibodies; EGPA = eosinophilic granulomatosis with polyangiitis; GPA = granulomatosis with polyangiitis; LoA = level of agreement; $\mathrm{MPA}=$ microscopic polyangiitis; $\mathrm{MPO}=$ myeloperoxidase; $\mathrm{PR} 3=$ proteinase -3 .

Scores: 1 =strongly disagree, 2 = disagree, 3 =undecided, $4=$ agree, $5=$ strongly agree.
The efficacy and safety of mepolizumab in EGPA were established in the MIRRA trial that recruited 136 patients with relapsing/refractory disease receiving stable oral glucocorticoids for 4 or more weeks (52). A total of $78 \%$ versus $32 \%$ of patients $(P<0.001)$ experienced clinical benefit (remission at any time, $50 \%$ or greater glucocorticoid dose reduction during weeks 48-52, or no EGPA relapses) in mepolizumab- and placebo-treated patients, respectively (67). Analyses of outcomes according to ANCA status were not performed because $<10 \%$ of the participants were ANCA positive at baseline. Mepolizumab seems to be a promising agent to control the disease activity and to spare glucocorticoids, although its ability to control vasculitis manifestations remains unclear (46).

Reslizumab is another IL-5neutralizing antibody currently approved for the treatment of severe eosinophilic asthma. In nine patients with EGPA with severe steroid-dependent eosinophilic asthma, 48 weeks of treatment with intravenous reslizumab was associated with a significant reduction in oral corticosteroid use. However, the authors suggested that reslizumab may be less effective in controlling extrapulmonary manifestations of EGPA, such as neuropathy (68). A therapeutic antibody to the IL-5 receptor benralizumab is the subject of a current clinical trial in EGPA (MANDARA) (ClinicalTrials.gov Identifier: NCT04157348).

In summary, ANCA positivity in patients with EGPA may signal a requirement for more intensive immunosuppressive therapy (e.g., addition of cyclophosphamide or rituximab) because vasculitis features may be present that are usually not sufficiently treated with glucocorticoids as monotherapy. However, ANCA positivity alone does not indicate a need for intensification of treatment. The efficacy and long-term safety of mepolizumab in MPO-ANCAassociated EGPA remains to be established. In the MIRRA trial that showed clear clinical benefit of IL-5 inhibition in EGPA, most patients were ANCA negative at baseline. At present, the presence or absence of MPO-ANCA should not be regarded as a barrier to administration of rituximab or mepolizumab, respectively, if considered necessary in the clinical context. 


\section{Box 1. Indications for ANCA testing in suspected EGPA}

To assure appropriate ANCA-test usage to support the diagnosis of EGPA, ANCA should be requested for patients with asthma or rhinosinusitis and blood eosinophilia if one or more of the following clinical features are present:

- Fever, weight loss, arthralgia, and/or myalgia in the presence of laboratory signs of inflammation

- Pulmonary infiltrates

- Sensory peripheral or motor neuropathy (including mononeuritis multiplex)

- Unexplained heart disease (e.g., arrhythmias or decreased left ventricular function)

- Urine nephritic sediment, a rising creatinine combined with hematuria or new-onset hematuria

- Alveolar hemorrhage

- Ischemic abdominal pain and otherwise unexplained gastrointestinal disorder

- Purpura or other skin rashes

\section{ANCA Monitoring}

The utility of serial ANCA measurements for predicting relapse of AAV remains controversial owing to contradictory results of clinical studies (69-71).

In three recent studies, serial ANCA testing in AAV was found to be useful in prediction of relapse in patients with renal and/or pulmonary vasculitis but not in patients with more limited disease (72-74). In 2015, an international task force representing the European League Against Rheumatism, the European Renal Association-European Dialysis and Transplant Association, and EUVAS concluded that neither persistence nor fourfold rise nor reappearance of ANCA should lead to a change in therapy but more frequent clinical assessment should be considered (70).

Of note, all studies that evaluated the clinical value of ANCA monitoring were restricted to patients with GPA and MPA, and it is possible that these results cannot be extrapolated to EGPA. Most studies in patients with EGPA did not provide data on repeat ANCA testing following immunosuppressive therapy. In the study by Keogh and Specks, $76 \%$ of patients with active EGPA were P-ANCA positive, whereas most of those tested during remission or after treatment initiation were ANCA negative (10). Serial data, although limited, indicated that ANCA status appeared to correlate with disease activity. In the study by Sinico and colleagues, ANCA were detected in only $10.8 \%$ of initially positive patients tested at the end of the follow-up period, and in $18.8 \%$ of patients tested at the time of a relapse (12), whereas Saku and colleagues showed that the first EGPA relapse was associated with ANCA positivity in $61.9 \%$ of patients who were ANCA positive at onset and in $12.9 \%$ of patients who initially were ANCA negative (30). It is postulated that immunosuppressive therapy usually results in seroconversion in MPOANCA-positive patients, and relapse of EGPA is usually associated with reoccurrence of MPO-ANCA $(75,76)$. However, limited evidence precludes firm conclusions.

Serial ANCA measurements can have some value in predicting relapses of GPA and MPA in patients with renal vasculitis and/or alveolar hemorrhage. In EGPA, monitoring of ANCA is only useful when MPO-ANCA was tested positive at disease onset. Repeat ANCA testing is recommended in patients with MPOANCA-positive EGPA because persistence, rise, or reappearance of ANCA may justify more frequent clinical assessment.

\section{Conclusions}

ANCA can be detected in approximately $30-35 \%$ of patients with EGPA and support a diagnosis of EGPA established on the clinical grounds. Given inconsistent results of IIF and established specificity of autoantibodies (MPO-ANCA in most cases), ANCA testing in EGPA should be performed as with GPA and MPA according to the revised 2017 consensus. Antigenspecific immunoassays are the preferred approach to detect ANCA for diagnosis of EGPA. ANCA status defines two distinctive but overlapping subsets of EGPA ("vasculitic" and "eosinophilic"), which may differ in outcomes and response to therapy. MPO-ANCA positivity in EGPA is associated with $H L A-D Q$ and a higher occurrence of vasculitis features, such as renal involvement, neuropathy, and skin vasculitis. MPO-ANCA negativity in EGPA lacks an HLA association and is associated with IRF1/IL5 and GPA33 and a higher occurrence of cardiomyopathy and lung manifestations. However, ANCA status taken on its own is neither sensitive nor specific enough to identify the vasculitis phenotype of EGPA and at present cannot guide treatment decisions.

All recommendations stated in Table 2 reached a high level of agreement. Only one expert voted against statements 5 and 6.

Author disclosures are available with the text of this article at www.atsjournals.org.

European EGPA Study Group members (not included in the list of authors): Fabian Arndt, Germany; Allyson Egan, United Kingdom; JeanEmmanuel Kahn, France; Anna Kernder, Germany; Alfred Mahr, France; Julian Mahrhold, Germany; Chiara Marvisi, Italy; Thomas Neumann, Switzerland; Domenico Prisco, Italy; Carlo Salvarani, Italy; Franco Schiavon, Italy; Arianna Troilo, Germany; Maria L. Urban, Italy; Nils Venhoff, Germany. 


\section{References}

1. Bossuyt X, Cohen Tervaert JW, Arimura Y, Blockmans D, Flores-Suárez LF, Guillevin L, et al. Position paper: revised 2017 international consensus on testing of ANCAs in granulomatosis with polyangiitis and microscopic polyangiitis. Nat Rev Rheumatol 2017;13:683-692.

2. Damoiseaux J, Csernok E, Rasmussen N, Moosig F, van Paassen $P$, Baslund B, et al. Detection of antineutrophil cytoplasmic antibodies (ANCAs): a multicentre European Vasculitis Study Group (EUVAS) evaluation of the value of indirect immunofluorescence (IIF) versus antigen-specific immunoassays. Ann Rheum Dis 2017;76: 647-653.

3. Csernok E, Damoiseaux J, Rasmussen N, Hellmich B, van Paassen $P$ Vermeersch $\mathrm{P}$, et al. Evaluation of automated multi-parametric indirect immunofluorescence assays to detect anti-neutrophil cytoplasmic antibodies (ANCA) in granulomatosis with polyangiitis (GPA) and microscopic polyangiitis (MPA). Autoimmun Rev 2016;15: 736-741.

4. Harrison DJ, Simpson R, Kharbanda R, Abernethy VE, Nimmo G. Antibodies to neutrophil cytoplasmic antigens in Wegener's granulomatosis and other conditions. Thorax 1989;44:373-377.

5. Tervaert JW, Goldschmeding R, Elema JD, Limburg PC, van der Giessen M, Huitema MG, et al. Association of autoantibodies to myeloperoxidase with different forms of vasculitis. Arthritis Rheum 1990;33:1264-1272.

6. Tervaert JW, Elema JD, Kallenberg CG. Clinical and histopathological association of 29kD-ANCA and MPO-ANCA. APMIS Supp/ 1990; 19:35.

7. Tervaert JW, Limburg PC, Elema JD, Huitema MG, Horst G, The TH, et al. Detection of autoantibodies against myeloid lysosomal enzymes: a useful adjunct to classification of patients with biopsy-proven necrotizing arteritis. Am J Med 1991;91:59-66.

8. Tervaert JW, Goldschmeding R, Elema JD, von dem Borne AE, Kallenberg CG. Antimyeloperoxidase antibodies in the Churg-Strauss syndrome. Thorax 1991;46:70-71.

9. Guillevin L, Cohen P, Gayraud M, Lhote F, Jarrousse B, Casassus P. Churg-Strauss syndrome: clinical study and long-term follow-up of 96 patients. Medicine (Baltimore) 1999;78:26-37.

10. Keogh KA, Specks U. Churg-Strauss syndrome: clinical presentation, antineutrophil cytoplasmic antibodies, and leukotriene receptor antagonists. Am J Med 2003;115:284-290.

11. Sablé-Fourtassou R, Cohen P, Mahr A, Pagnoux C, Mouthon L, Jayne D, et al.; French Vasculitis Study Group. Antineutrophil cytoplasmic antibodies and the Churg-Strauss syndrome. Ann Intern Med 2005; 143:632-638.

12. Sinico RA, Di Toma L, Maggiore U, Bottero P, Radice A, Tosoni C, et al. Prevalence and clinical significance of antineutrophil cytoplasmic antibodies in Churg-Strauss syndrome. Arthritis Rheum 2005;52: 2926-2935.

13. Cohen $P$, Pagnoux $C$, Mahr A, Arène JP, Mouthon L, Le Guern V, et al.; French Vasculitis Study Group. Churg-Strauss syndrome with poor-prognosis factors: a prospective multicenter trial comparing glucocorticoids and six or twelve cyclophosphamide pulses in forty-eight patients. Arthritis Rheum 2007;57:686-693.

14. Ribi C, Cohen P, Pagnoux C, Mahr A, Arène JP, Lauque D, et al.; French Vasculitis Study Group. Treatment of Churg-Strauss syndrome without poor-prognosis factors: a multicenter, prospective, randomized, open-label study of seventy-two patients. Arthritis Rheum 2008;58:586-594.

15. Baldini C, Della Rossa A, Grossi S, Catarsi E, Talarico R, d'Ascanio A, et al. Churg-Strauss syndrome: outcome and long-term follow-up of 38 patients from a single Italian centre [in Italian]. Reumatismo 2009; 61:118-124.

16. Neumann T, Manger B, Schmid M, Kroegel C, Hansch A, Kaiser WA, et al. Cardiac involvement in Churg-Strauss syndrome: impact of endomyocarditis. Medicine (Baltimore) 2009;88:236-243.

17. Dennert RM, van Paassen P, Schalla S, Kuznetsova T, Alzand BS, Staessen JA, et al. Cardiac involvement in Churg-Strauss syndrome. Arthritis Rheum 2010;62:627-634.
18. Healy B, Bibby S, Steele R, Weatherall M, Nelson H, Beasley R. Antineutrophil cytoplasmic autoantibodies and myeloperoxidase autoantibodies in clinical expression of Churg-Strauss syndrome. J Allergy Clin Immunol 2013;131:571-6.e1, 6.

19. Samson M, Puéchal X, Devilliers H, Ribi C, Cohen P, Stern M, et al.; French Vasculitis Study Group. Long-term outcomes of 118 patients with eosinophilic granulomatosis with polyangiitis (Churg-Strauss syndrome) enrolled in two prospective trials. J Autoimmun 2013;43: 60-69.

20. Comarmond C, Pagnoux C, Khellaf M, Cordier JF, Hamidou M, Viallard JF, et al.; French Vasculitis Study Group. Eosinophilic granulomatosis with polyangiitis (Churg-Strauss): clinical characteristics and long-term followup of the 383 patients enrolled in the French Vasculitis Study Group cohort. Arthritis Rheum 2013;65: 270-281.

21. Sada KE, Amano K, Uehara R, Yamamura M, Arimura Y, Nakamura Y, et al.; Research Committee on Intractable Vasculitides, the Ministry of Health, Labour, Welfare of Japan. A nationwide survey on the epidemiology and clinical features of eosinophilic granulomatosis with polyangiitis (Churg-Strauss) in Japan. Mod Rheumatol 2014;24: 640-644.

22. Moosig F, Bremer JP, Hellmich B, Holle JU, Holl-Ulrich K, Laudien M, et al. A vasculitis centre based management strategy leads to improved outcome in eosinophilic granulomatosis and polyangiitis (Churg-Strauss, EGPA): monocentric experiences in 150 patients. Ann Rheum Dis 2013;72:1011-1017.

23. Sokolowska BM, Szczeklik WK, Wludarczyk AA, Kuczia PP, Jakiela BA Gasior JA, et al. ANCA-positive and ANCA-negative phenotypes of eosinophilic granulomatosis with polyangiitis (EGPA): outcome and long-term follow-up of 50 patients from a single Polish center. Clin Exp Rheumatol 2014;32(Suppl 82):S41-S47.

24. Dunogué B, Terrier B, Cohen P, Marmursztejn J, Legmann $P$, Mouthon L, et al.; French Vasculitis Study Group. Impact of cardiac magnetic resonance imaging on eosinophilic granulomatosis with polyangiitis outcomes: a long-term retrospective study on 42 patients. Autoimmun Rev 2015;14:774-780.

25. Hazebroek MR, Kemna MJ, Schalla S, Sanders-van Wijk S, Gerretsen $\mathrm{SC}$, Dennert R, et al. Prevalence and prognostic relevance of cardiac involvement in ANCA-associated vasculitis: eosinophilic granulomatosis with polyangiitis and granulomatosis with polyangiitis. Int $J$ Cardiol 2015;199:170-179.

26. Durel CA, Berthiller J, Caboni S, Jayne D, Ninet J, Hot A. Long-term followup of a multicenter cohort of 101 patients with eosinophilic granulomatosis with polyangiitis (Churg-Strauss). Arthritis Care Res (Hoboken) 2016;68:374-387.

27. Cottin V, Bel E, Bottero P, Dalhoff K, Humbert M, Lazor R, et al.; Groupe d'Etudes et de Recherche sur les Maladies Orphelines Pulmonaires (GERM"O"P). Revisiting the systemic vasculitis in eosinophilic granulomatosis with polyangiitis (Churg-Strauss): a study of 157 patients by the Groupe d'Etudes et de Recherche sur les Maladies Orphelines Pulmonaires and the European Respiratory Society Taskforce on eosinophilic granulomatosis with polyangiitis (Churg-Strauss). Autoimmun Rev 2017;16: $1-9$.

28. Solans-Laqué R, Fraile G, Rodriguez-Carballeira M, Caminal L, Castillo MJ, Martínez-Valle F, et al.; Spanish Registry of systemic vasculitis (REVAS) from the Autoimmune Diseases Study Group (GEAS) of the Spanish Society of Internal Medicine (SEMI). Clinical characteristics and outcome of Spanish patients with ANCA-associated vasculitides: impact of the vasculitis type, ANCA specificity, and treatment on mortality and morbidity. Medicine (Baltimore) 2017;96: e6083.

29. Tsurikisawa N, Oshikata C, Kinoshita A, Tsuburai T, Saito H. Longterm prognosis of 121 patients with eosinophilic granulomatosis with polyangiitis in Japan. $J$ Rheumatol 2017;44:1206-1215.

30. Saku A, Furuta S, Hiraguri M, Ikeda K, Kobayashi Y, Kagami SI, et al. Longterm outcomes of 188 Japanese patients with eosinophilic granulomatosis with polyangiitis. J Rheumatol 2018; 45:1159-1166. 
31. Schroeder JW, Folci M, Losappio LM, Chevallard M, Sinico RA, Mirone $\mathrm{C}$, et al. Anti-neutrophil cytoplasmic antibodies positivity and antileukotrienes in eosinophilic granulomatosis with polyangiitis: a retrospective monocentric study on 134 Italian patients. Int Arch Allergy Immunol 2019;180:64-71.

32. Lyons PA, Peters JE, Alberici F, Liley J, Coulson RMR, Astle W, et al.; European Vasculitis Genetics Consortium. Genome-wide association study of eosinophilic granulomatosis with polyangiitis reveals genomic loci stratified by ANCA status. Nat Commun 2019;10:5120.

33. van Beers JJBC, Vanderlocht J, Roozendaal C, Damoiseaux J. Detection of anti-neutrophil cytoplasmic antibodies (ANCA) by indirect immunofluorescence. Methods Mol Biol 2019;1901:47-62.

34. Shida H, Nakazawa D, Tateyama Y, Miyoshi A, Kusunoki Y, Hattanda F, et al. The presence of anti-lactoferrin antibodies in a subgroup of eosinophilic granulomatosis with polyangiitis patients and their possible contribution to enhancement of neutrophil extracellular trap formation. Front Immunol 2016;7:636.

35. Mukherjee M, Thomas SR, Radford K, Dvorkin-Gheva A, Davydchenko $\mathrm{S}$, Kjarsgaard M, et al. Sputum antineutrophil cytoplasmic antibodies in serum antineutrophil cytoplasmic antibody-negative eosinophilic granulomatosis with polyangiitis. Am J Respir Crit Care Med 2019; 199:158-170.

36. Foucher $P$, Heeringa $P$, Petersen AH, Huitema MG, Brouwer E, Tervaert $\mathrm{JW}$, et al. Antimyeloperoxidase-associated lung disease: an experimental model. Am J Respir Crit Care Med 1999;160:987-994.

37. Xiao H, Heeringa P, Hu P, Liu Z, Zhao M, Aratani Y, et al. Antineutrophil cytoplasmic autoantibodies specific for myeloperoxidase cause glomerulonephritis and vasculitis in mice. J Clin Invest 2002;110: 955-963.

38. Dejaco C, Oppl B, Monach P, Cuthbertson D, Carette S, Hoffman G, et al. Serum biomarkers in patients with relapsing eosinophilic granulomatosis with polyangiitis (Churg-Strauss). PLoS One 2015; 10:e0121737.

39. Zwerina J, Bach C, Martorana D, Jatzwauk M, Hegasy G, Moosig F, et al. Eotaxin-3 in Churg-Strauss syndrome: a clinical and immunogenetic study. Rheumatology (Oxford) 2011;50:1823-1827.

40. Vaglio A, Strehl JD, Manger B, Maritati F, Alberici F, Beyer C, et al. IgG4 immune response in Churg-Strauss syndrome. Ann Rheum Dis 2012; 71:390-393.

41. Zagvozdkina ES, Moiseev SV, Novikov PI. Eotaxin-3 as a biomarker of activity in established eosinophilic granulomatosis with polyangiitis. $J$ Rheumatol 2016;43:2082-2083.

42. Chaigne B, Terrier B, Thieblemont N, Witko-Sarsat V, Mouthon L. Dividing the Janus vasculitis? Pathophysiology of eosinophilic granulomatosis with polyangitis. Autoimmun Rev 2016:15:139-145.

43. Nakazawa D, Shida H, Tomaru U, Yoshida M, Nishio S, Atsumi T, et al. Enhanced formation and disordered regulation of NETs in myeloperoxidase-ANCA-associated microscopic polyangiitis. J Am Soc Nephrol 2014;25:990-997.

44. Sangaletti S, Tripodo C, Chiodoni C, Guarnotta C, Cappetti B, Casalini $\mathrm{P}$, et al. Neutrophil extracellular traps mediate transfer of cytoplasmic neutrophil antigens to myeloid dendritic cells toward ANCA induction and associated autoimmunity. Blood 2012;120: 3007-3018.

45. Nishi R, Koike H, Ohyama K, Fukami Y, Ikeda S, Kawagashira $Y$, et al. Differential clinicopathologic features of EGPA-associated neuropathy with and without ANCA. Neurology 2020;94:e1726e1737.

46. Berti A, Boukhlal S, Groh M, Cornec D. Eosinophilic granulomatosis with polyangiitis: the multifaceted spectrum of clinical manifestations at different stages of the disease. Expert Rev Clin Immunol 2020;16: 51-61.

47. Lanham JG, Elkon KB, Pusey CD, Hughes GR. Systemic vasculitis with asthma and eosinophilia: a clinical approach to the Churg-Strauss syndrome. Medicine (Baltimore) 1984;63:65-81.

48. Masi AT, Hunder GG, Lie JT, Michel BA, Bloch DA, Arend WP, et al. The American College of Rheumatology 1990 criteria for the classification of Churg-Strauss syndrome (allergic granulomatosis and angiitis). Arthritis Rheum 1990;33:1094-1100.
49. Watts R, Lane S, Hanslik T, Hauser T, Hellmich B, Koldingsnes W, et al. Development and validation of a consensus methodology for the classification of the ANCA-associated vasculitides and polyarteritis nodosa for epidemiological studies. Ann Rheum Dis 2007;66: 222-227.

50. Jennette JC, Falk RJ, Bacon PA, Basu N, Cid MC, Ferrario F, et al. 2012 revised international Chapel Hill consensus conference nomenclature of vasculitides. Arthritis Rheum 2013;65:1-11.

51. Groh M, Pagnoux C, Baldini C, Bel E, Bottero P, Cottin V, et al. Eosinophilic granulomatosis with polyangiitis (Churg-Strauss) (EGPA) Consensus Task Force recommendations for evaluation and management. Eur J Intern Med 2015;26: 545-553.

52. Wechsler ME, Akuthota P, Jayne D, Khoury P, Klion A, Langford CA, et al.; EGPA Mepolizumab Study Team. Mepolizumab or placebo for eosinophilic granulomatosis with polyangiitis. N Engl J Med 2017; 376:1921-1932.

53. Robson J, Grayson P, Ponte C, Suppiah R, Craven A, Khalid S, et al. Draft classification criteria for the ANCA-associated vasculitides. Ann Rheum Dis 2018;77(Suppl 2):60.2-61.

54. Moiseev S, Coher Tervaert JW, Arimura Y, Bogdanos DP, Csernok E, Damoiseaux J, et al. International consensus on ANCA testing beyond systemic vasculitis. Autoimmun Rev 2020;19:102618.

55. Zhang J, Zhang HY, Chen SZ, Huang JY. Anti-neutrophil cytoplasmic antibodies in cholesterol embolism: a case report and literature review. Exp Ther Med 2016;12:1012-1018.

56. Delen S, Boonen A, Landewé R, Kroon AA, van der Linden S, Tervaert JW. An unusual case of ANCA positive disease. Ann Rheum Dis 2003;62:780-781.

57. Moiseev S, Novikov P. Five Factor Score in patients with eosinophilic granulomatosis with polyangiitis (Churg-Strauss; EGPA): to use or not to use? Ann Rheum Dis 2014;73:e12.

58. Jones RB, Tervaert JWC, Hauser T, Luqmani R, Morgan MD, Peh CA, et al.; European Vasculitis Study Group. Rituximab versus cyclophosphamide in ANCA-associated renal vasculitis. $N$ Engl $J$ Med 2010;363:211-220.

59. Stone JH, Merkel PA, Spiera R, Seo P, Langford CA, Hoffman GS, et al.; RAVE-ITN Research Group. Rituximab versus cyclophosphamide for ANCA-associated vasculitis. N Engl J Med 2010;363:221-232.

60. Guillevin L, Pagnoux C, Karras A, Khouatra C, Aumaître O, Cohen P, et al.; French Vasculitis Study Group. Rituximab versus azathioprine for maintenance in ANCA-associated vasculitis. N Engl J Med 2014; $371: 1771-1780$.

61. Novikov P, Moiseev S, Smitienko I, Zagvozdkina E. Rituximab as induction therapy in relapsing eosinophilic granulomatosis with polyangiitis: a report of 6 cases. Joint Bone Spine 2016; 83:81-84.

62. Mohammad AJ, Hot A, Arndt F, Moosig F, Guerry MJ, Amudala N, et al. Rituximab for the treatment of eosinophilic granulomatosis with polyangiitis (Churg-Strauss). Ann Rheum Dis 2016;75: 396-401.

63. Teixeira V, Mohammad AJ, Jones RB, Smith R, Jayne D. Efficacy and safety of rituximab in the treatment of eosinophilic granulomatosis with polyangiitis. $R M D$ Open 2019;5:e000905.

64. Casal Moura M, Berti A, Keogh KA, Volcheck GW, Specks U, Baqir M. Asthma control in eosinophilic granulomatosis with polyangiitis treated with rituximab. Clin Rheumatol 2020;39:1581-1590.

65. Pepper RJ, Fabre MA, Pavesio C, Gaskin G, Jones RB, Jayne D, et al. Rituximab is effective in the treatment of refractory ChurgStrauss syndrome and is associated with diminished T-cell interleukin-5 production. Rheumatology (Oxford) 2008;47: 1104-1105.

66. Emmi G, Rossi GM, Urban ML, Silvestri E, Prisco D, Goldoni M, et al. Scheduled rituximab maintenance reduces relapse rate in eosinophilic granulomatosis with polyangiitis. Ann Rheum Dis 2018; 77:952-954.

67. Steinfeld J, Bradford ES, Brown J, Mallett S, Yancey SW, Akuthota P, et al. Evaluation of clinical benefit from treatment with mepolizumab for patients with eosinophilic granulomatosis with polyangiitis. J Allergy Clin Immunol 2019;143:2170-2177. 
68. Kent BD, d'Ancona G, Fernandes M, Green L, Roxas C, Thomson L, et al. Oral corticosteroid-sparing effects of reslizumab in the treatment of eosinophilic granulomatosis with polyangiitis. ERJ Open Res 2020;6:00311-02019.

69. Verstockt B, Bossuyt X, Vanderschueren S, Blockmans D. There is no benefit in routinely monitoring ANCA titres in patients with granulomatosis with polyangiitis. Clin Exp Rheumatol 2015;33(Suppl 89):S-72-S-76.

70. Yates M, Watts RA, Bajema IM, Cid MC, Crestani B, Hauser T, et al. EULAR/ERA-EDTA recommendations for the management of ANCA-associated vasculitis. Ann Rheum Dis 2016;75: 1583-1594.

71. Kemna MJ, van Paassen P, Damoiseaux JGMC, Cohen Tervaert JW. Maintaining remission in patients with granulomatosis with polyangiitis or microscopic polyangiitis: the role of ANCA. Expert Opin Orphan Drugs 2017;5:207-218.

72. Kemna MJ, Damoiseaux J, Austen J, Winkens B, Peters J, van Paassen $P$, et al. ANCA as a predictor of relapse: useful in patients with renal involvement but not in patients with nonrenal disease. J Am Soc Nephrol 2015;26:537-542.
73. Yamaguchi M, Ando M, Kato S, Katsuno T, Kato N, Kosugi T, et al. Increase of antimyeloperoxidase antineutrophil cytoplasmic antibody (ANCA) in patients with renal ANCA-associated vasculitis: association with risk to relapse. J Rheumatol 2015;42:1853-1860.

74. Fussner LA, Hummel AM, Schroeder DR, Silva F, Cartin-Ceba R, Snyder MR, et al.; Rituximab in ANCA-Associated Vasculitis-Immune Tolerance Network Research Group. Factors determining the clinical utility of serial measurements of antineutrophil cytoplasmic antibodies targeting proteinase 3. Arthritis Rheumatol 2016;68:1700-1710.

75. Bremer JP, Csernok E, Holle J, Gross WL, Moosig F. Getting rid of MPO-ANCA: a matter of disease subtype. Rheumatology (Oxford) 2013;52:752-754.

76. Watanabe H, Sada KE, Matsumoto Y, Harigai M, Amano K, Dobashi H, et al.; Japan Research Committee of the Ministry of Health, Labour, and Welfare for Intractable Vasculitis, and the Research Committee of Intractable Renal Disease of the Ministry of Health, Labour, and Welfare of Japan. Association between reappearance of myeloperoxidase-antineutrophil cytoplasmic antibody and relapse in antineutrophil cytoplasmic antibody-associated vasculitis: subgroup analysis of nationwide prospective cohort studies. Arthritis Rheumatol 2018;70:1626-1633. 